\title{
High-Speed Road Measurement Systems: Integration of Pavement and General Mapping Laser Technologies
}

\author{
Enea Sogno and Michele Mori \\ Sina S.p.A., Milan 20135, Italy
}

\begin{abstract}
Performing surveys on a single section of a road is quite different from that on a road network. In the first case, the need for performance characteristics of the pavement may be of service to rehabilitation. Surveying a road network, instead, aims to establish service levels, identify deficiencies and priorities and define budgets for maintenance. This challenge needs a methodological approach in order to widen the action plan and optimize the cost-benefit analysis of each asset through the use of preventive maintenance policies. High-speed monitoring systems on the market were specialized towards the necessity of measuring specific performance of the pavement or mapping land (data inventory). The technological development of instrumentations such as inertial systems and synchronizers has enabled the integration of different devices and the achievement of the best correlation of data. This is the case of the LARAN (laser road analyzer), whose helpfulness will be presented within a case study in which geometrical data and surface characteristic information — cracks, texture, profiles-were obtained through a unique survey. The cross-analysis resulted efficient for safety purposes and also for the identification of "black points" corresponding to boundary operative conditions of the road with respect to technical standards and recommendations.
\end{abstract}

Key words: Pavement surface properties, data quality, monitoring and inspection, road and airfield safety, case studies.

\section{Introduction}

The high efficiency surveying system LARAN (laser road analyzer) combines the Pavemetrics LCMS@ with the Optech Lynx Mobile Mapper. The result of the integration is that a unique equipment produces big advantages for pavement monitoring and controls (any infrastructure works, and street furniture), for PMS applications and also for safety analysis purposes based on the geometrical and operative conditions of the infrastructure, especially when accessory information from other surveys is available.

Surveys performed with the LARAN comply with international standards' requirements and may provide a great number of detailed information which can be managed with the help of appropriate software. Mainly, high-resolution 3D imagery of the pavement surface can be achieved simultaneously with laser scans, thus improving the interpretation and

Corresponding author: Enea Sogno, Ph.D., civil engineer, research fields: PMS and pavement design. comprehensibility of surface distress, and the description of the surroundings coupled with 3D imagery can be obtained as an output of the operation of laser sensors.

It is important to understand that georeferenced data sets coming out from post processing (i.e. spherical imagery, point clouds, pavement 3D bit-maps, longitudinal and transverse profiles, rutting, macrotexture, cracking and other pavement defects) allow infrastructure managers not only to deepen their confidence with the road network and have a complete control of the needs for maintenance but also to get the amount of distress on the pavement surface and represent it by means of synthetic indexes such as the PCI (see Refs. [1, 2]).

On the base of the above-mentioned information, a case study has been developed to highlight the importance of guaranteeing suitable operative conditions of the carriageway and reducing potential risks for safety. As a result, considerations have been formulated on technical concerns which directly affect 
management strategies and maintenance policies.

\section{Method and Materials}

The LARAN is a high efficiency equipment intended to help infrastructure managers and maintenance operators to improve their actions and services on quality assurance.

This new generation device comes from the integration of the LCMS technology held by Pavemetrics with a high precision Laser Mobile Mapping device. By this way, the LARAN is a powerful high-efficiency laser technology that gives the opportunity of detailing the condition of civil infrastructures and of their pavement surface.

\subsection{LARAN System: Structure}

From an operational viewpoint, the system is based on a controller (the core) that governs the activity of several laser and lidar sensors being able to detect different targets for considerable distances around the machine. Lots of characteristics of these targets can be easily described in most ways through the elaboration of their response to signals, based on a very precise 3D data profiles collection and visualization plus the measurement capability they offer both in the space and upon the pavement surface.

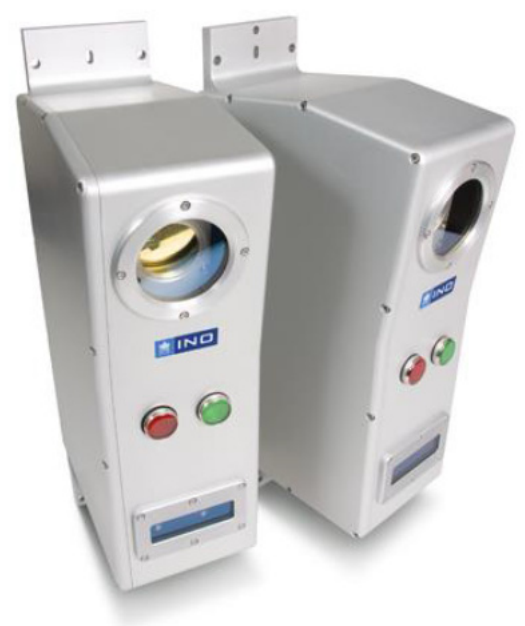

(a)
The installed sensors have been fully integrated with high performance traditional and spherical cameras, in order to improve the representation of the information belonging to the survey (see Figs. 1a and 1b).

A powerful console allows the operator first to successfully finalize data acquisition, then to process them by sorting and grouping all the obtained information in order to constitute a well-organized database. Finally, data reporting follows the selection of the information to be managed inside the same scalable database.

\subsection{LARAN System: Software}

The LARAN reveals its potential especially with the support of dedicated software, since the acquired data result very abundant, time-consuming and quite complex to manage due to the quantity and precision of information they can represent. The solution offered by Pavemetics has then been improved so that further and more direct indicators could be obtained as a result of processing and post-processing operations. By this way, the automatic generation of all data sets being necessary for design and maintenance purposes and the setup of the involved assets can be easily consulted in the GIS environment (see Fig. 2), and the selection.

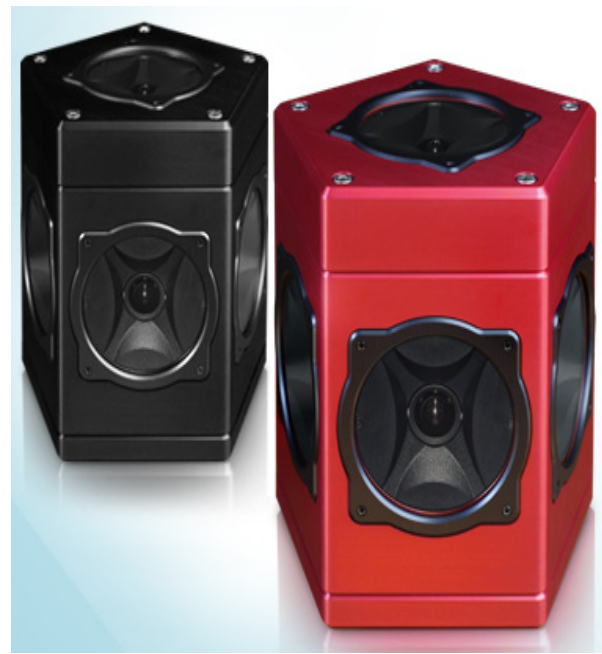

(b)

Fig. 1 LCMS laser sensors (a) and mounted spherical cameras (b). 


\section{General Mapping Laser Technologies}

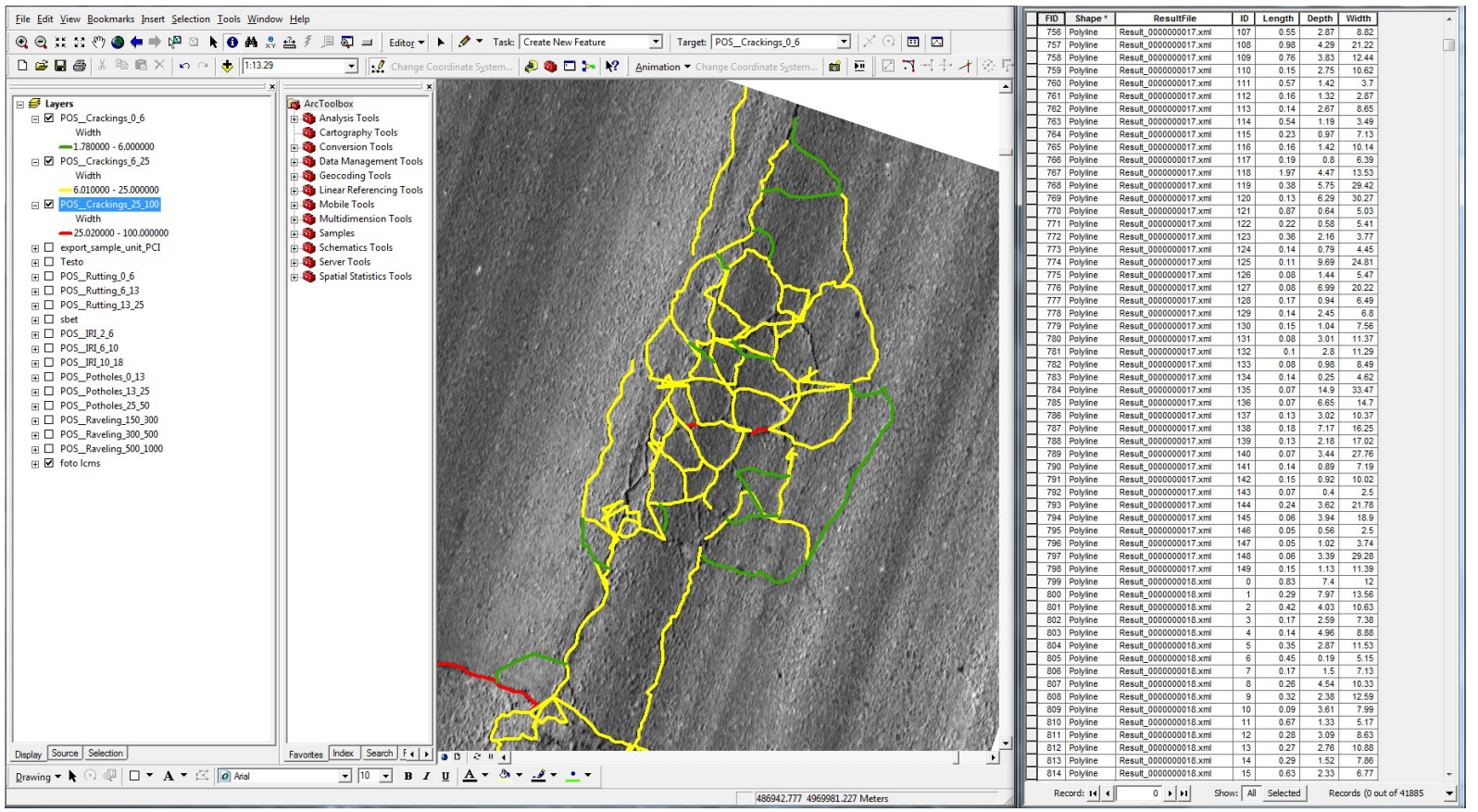

Fig. 2 Pavement surface distress analysis and visualization in GIS environment.

\subsection{Survey Specifications}

With the intention of evaluating the usefulness of the system to the needs of infrastructure managers, a $5.270 \mathrm{~km}$ long test section has been surveyed with the LARAN and the analysis of the information acquired on that has been then performed. In the following sub-sections a detailed description of the site, data collection and results will be provided with the effort of illustrating the potential of such technology not only for maintenance purposes but also for safety analyses on road networks, especially in the case of the availability of complementary parameters (i.e. skid resistance, roughness).

\subsubsection{Infrastructure and Materials}

The selected test site is part of an Italian motorway heavily trafficked due to the high development of logistics in the regions it connects. A $5.270 \mathrm{~km}$ long section (one direction) has been chosen in order to dispose of variable track characteristics and speed modifications during the analysis of vehicles' operation in the conditions established from survey results.

With regard to the infrastructure, it includes tunnels, bridges and opencast sections. According to that, the carriageway reveals different arrangements and visual perspectives due to which the transversal and longitudinal profiles to be considered for evaluating roughness and potential risks produced by excessive speed assume different shapes. Figs. $3 a$ and $3 b$, and Table 1, illustrate the resume of altimetry and planimetry on the test section.

Focusing on the pavement, its surface shows the existence of a close-graded asphalt concrete having been spread out in different times along the section. The drainage of meteoric waters constantly occurs upon the wearing course.

2.3.2 Collected Data and Supplementary Information

Most data used for processing purposes have been directly provided by the LARAN, even though additional indicators being available at the moment of the survey have been used and merged in a unique dataset containing all the necessary information. Among them, it is important to cite at least the skid rates on both wheel-paths of the slow lane which were previously documented by the road manager.

During the analysis three main groups of information were considered: 


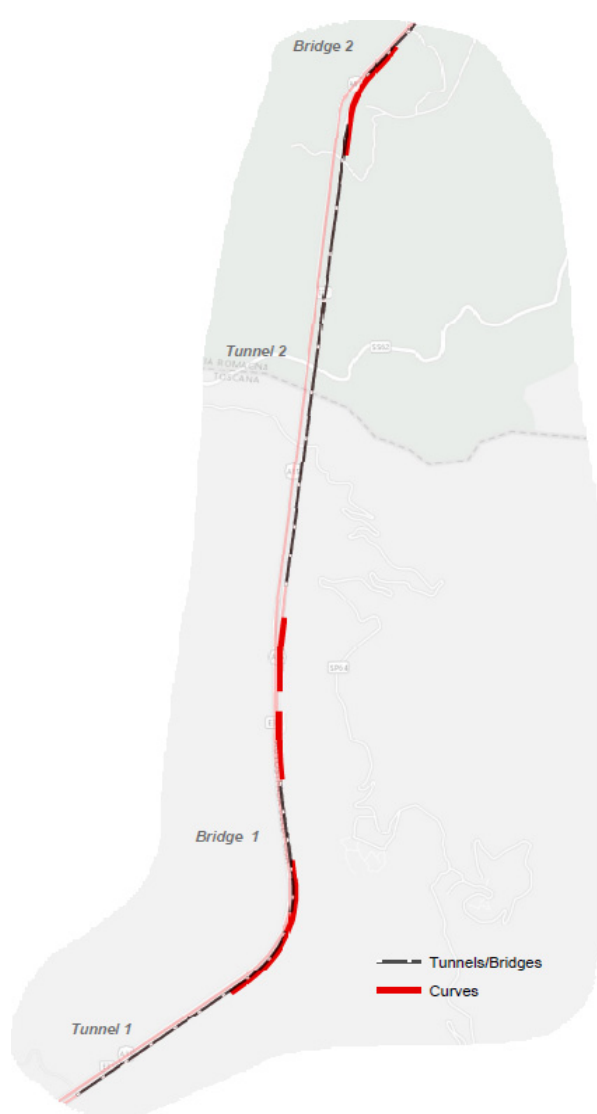

(a)

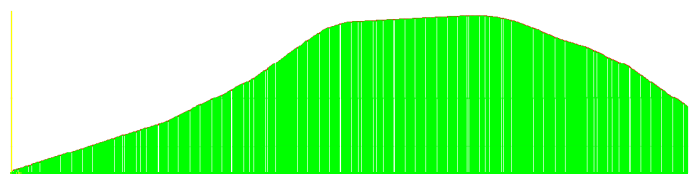

(b)

Fig. 3 Test site geometry (a) and vertical profile (b).

Table 1 Principal numbers of the test site.

\begin{tabular}{ll}
\hline ID & Number \\
\hline Tunnels & 2 \\
Bridges & 2 \\
Opencast transitions & 3 \\
Right curves & 3 \\
Left curves & 1 \\
Straights & 4 \\
Side slope inversion & 1 \\
Lanes & 2 \\
\hline
\end{tabular}

(1) Geometrical information of the infrastructure and the carriageway (see Fig. 4), from point clouds (i.e. carriageway limits, profiles and transverse sections, start/end of tunnels and bridges, etc.);

(2) Functional properties of the pavement surface, from 3D pavement images and accessory surveys (macrotexture, longitudinal roughness, lane markings, skid resistance);

(3) Operating conditions of the track, from documents provided by the road manager (min. and max. design speed, operating speed, min. and max. longitudinal and transverse slope in straights and curves, vertical and horizontal curve radii, existence of safety barriers, etc.).

\section{Results and Discussion}

The assessment of road conditions at the time of the survey leads to the individuation of sections that appear deficient if compared to the rest of the track. According to that, the definition of the activities which 


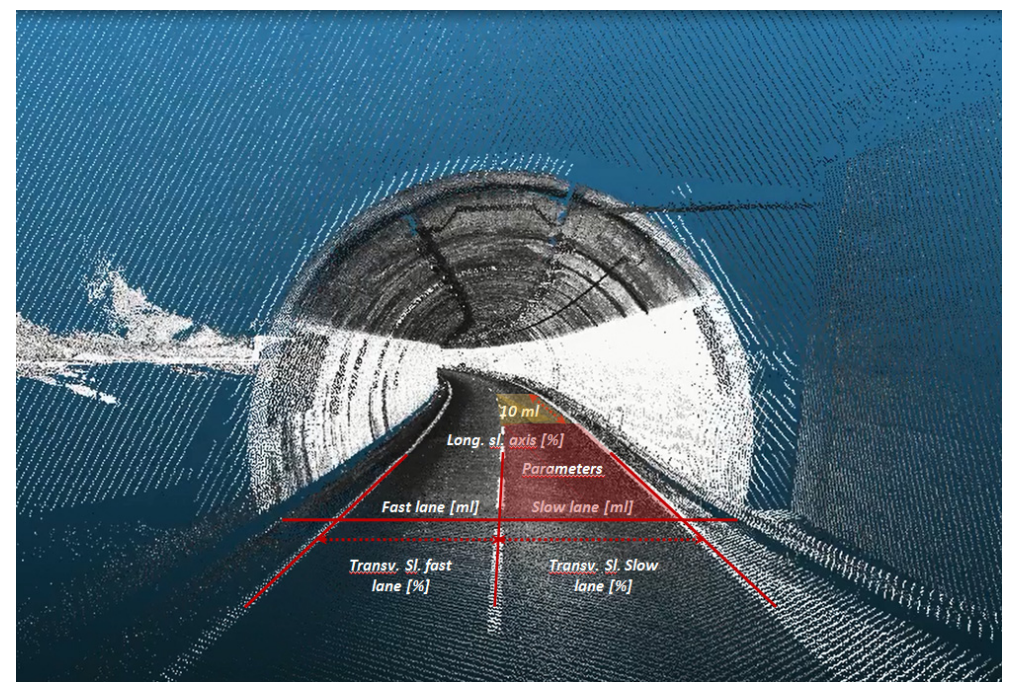

Fig. 4 Particular of tunnel 1 from LMM data processing (point clouds).

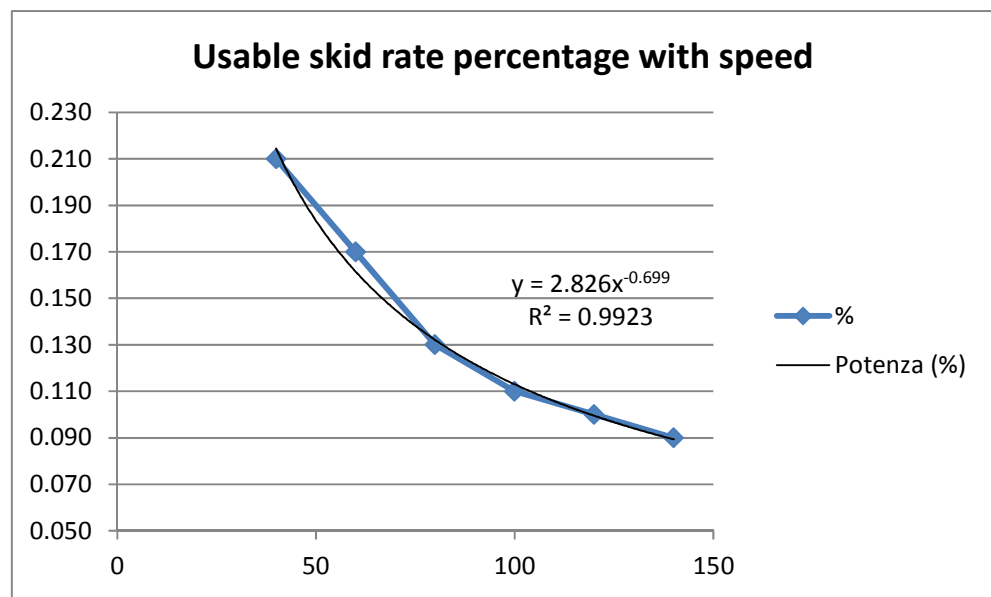

Fig. 5 Relationship between the effective available skid rate and the operating speed according to Ref. [3] (measured vs. estimated values).

could positively affect traffic operation and safety and the estimation of pavement performance after maintenance of the interested sections have been pursued also by taking into account performance modifications due to the geometry of the carriageway and the operating speed.

The above mentioned process has been developed not only focusing on the functional properties as stated by laser and complementary surveys but also looking for the relationship between them and the geometry of the carriageway. By doing this, a dynamic analysis has been effectively carried out since each of the involved parameters intervenes in the process not as a unique indicator but with a meaning that depends on all the other factors that were included in the process. For instance, macrotexture and longitudinal roughness of the pavement surface have been treated with its skid resistance, the operating speed and the slopes of the considered section, thus providing more complex indicators in the suggestion for maintenance.

Three different scenarios have been assumed for simulation purposes, selecting operating speeds of 80 $\mathrm{km} / \mathrm{h}, 90 \mathrm{~km} / \mathrm{h}$ and $100 \mathrm{~km} / \mathrm{h}$.

\subsection{Pavement Performance}

With respect to the performance of the pavement surface, the existing level has been established by 
evaluating the results of laser surveys compared with thresholds to skid values and the macrotexture (longitudinal roughness has been treated excluding joints and pavement transitions, as in Ref. [4]) that can be found in the literature (see Refs. $[5,6]$ ) and technical specifications (see Refs. [7, 8]):

$$
\begin{aligned}
& \text { Skid }_{\mathrm{ref}}>\text { Skid }_{\text {min }} \\
& \text { Mtex }_{\mathrm{ref}}>\text { Mtex }_{\min } \\
& \text { Unev }_{\mathrm{ref}}<\text { Unev }_{\text {max }}
\end{aligned}
$$

It should be noticed that skid values to be assumed as thresholds depend on the type of wearing course, on the transverse slope of the carriageway and on the recommended operating speed in the section. As well, macrotexture values depend on the type of wearing course, on the transverse slope of the carriageway and on the minimum skid value resulting from Eq. (1).

\subsection{Road Geometry}

Geometry check has been related to the carriageway width and slopes, the differential slope between the slow lane and the fast lane, the vertical and horizontal curve radii, and the length of the curve sections (see Fig. 6). With reference to this, the following conditions have been analysed by referring to national and European technical standards (see Refs. [3, 9]):

$$
\text { Width }_{\mathrm{car}}>\text { Width }_{\min }
$$

$$
\begin{aligned}
& \text { Slope }_{\mathrm{lane}}>\text { Slope }_{\mathrm{min}} \\
& \operatorname{Rad}_{\mathrm{ref}}>\operatorname{Rad}_{\mathrm{min}} \\
& \text { Leng }_{\mathrm{ref}}>\text { Leng }_{\mathrm{min}}
\end{aligned}
$$

Eq. (4) has not been considered for special sections such as tunnels, where the limits of the carriageway must fit the physical border of the infrastructure in any case. Eq. (5) has been implemented with reference to the longitudinal axis of each lane. Coherence between the slopes of the slow and the fast lane has been searched based on reference values coming out from Eq. (5).

\subsection{Operation and Maintenance Activities}

From an operating point of view, the unique control being performed is whether safety barriers exist or not at the outer border of the carriageway.

Suggested actions have been processed in order to allow the road manager to prevent the occurrence of accidents as in Ref. [10] first by reducing the operating speed and installing protection devices where necessary, thus avoiding major costs from pavement repair and carriageway modifications. In addition to that, a set of treatments for the pavement surface has been considered to make it possible to improve skid and macrotexture characteristics and to re-profile the lane, where necessary.

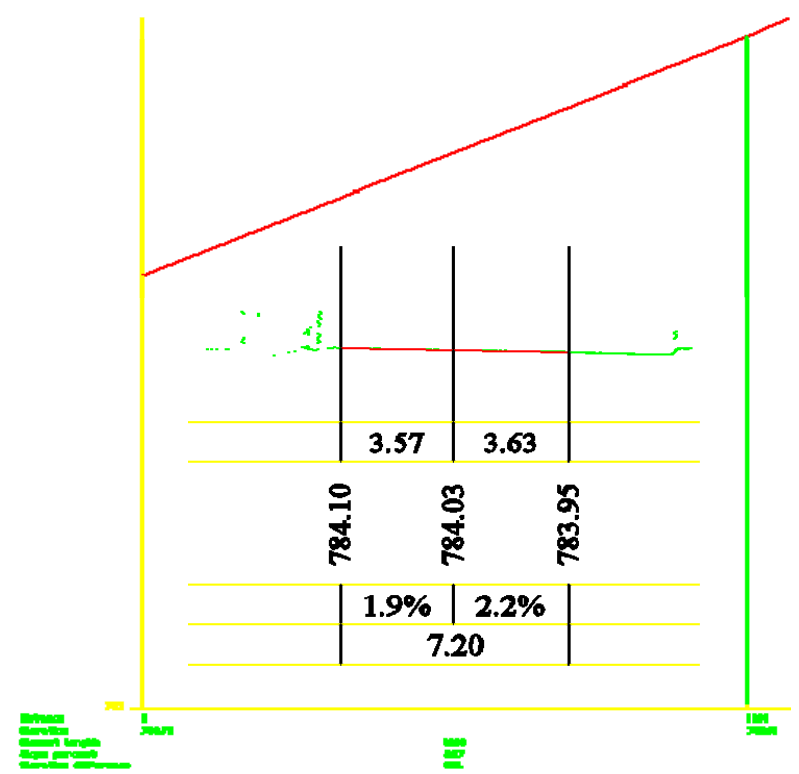

Fig. 6 Example of geometrical data organization. 


\subsection{Outcomes}

From a technical viewpoint, the above described approach is brought to the identification of some portions of the track denoting relevant deficiencies with respect to surface characteristics and the organization of the platform (see Fig. 3 to check the location of the sections listed below). A 3D simulation based on point clouds has been implemented too, in order to assess operation in these regions at the speeds indicated before.

- End of Tunnel 1: low skid and macrotexture values from $0.800 \mathrm{~km}$ to $1.470 \mathrm{~km}$, no safety barriers in the approach to Bridge 1 and positive difference between the transverse slope of the fast lane and the slow lane, with left curve.

- Bridge 1: no minimum transverse slopes from $1.470 \mathrm{~km}$ to $2.610 \mathrm{~km}$.

- Tunnel 2: low skid and macrotexture values from $3.050 \mathrm{~km}$ to $3.270 \mathrm{~km}$ (straight) and from $4.340 \mathrm{~km}$ to $5.270 \mathrm{~km}$ (right curve).

- Bridge 2: no minimum transverse slopes from

\section{$5.150 \mathrm{~km}$ to $5.270 \mathrm{~km}$.}

Figs. 7 and 8 resume these findings by emphasizing the kinetic perspective, thus giving suggestions for further analyses and considerations based on the inertial parameters which define the behavior of advancing vehicles. It could be underlined that the very detailed level of information which was possible owing to the exceptional resolution of the scanner system and the high frequency of measurements produced by accessory surveying devices allowed to properly identify risks suffered from traffic during operation, so that safety conditions could be indirectly treated and focused on.

Figs. 9-12 further describe the correspondence of the kinetic phenomena listed before with data coming out from surveys, among which roll and pitch are particularly relevant when compared to the transverse slope of the carriageway. It has been found out, in particular, that a strong correlation between transverse slopes and lateral accelerations of the vehicle exists, independently of road geometry $\left(\mathrm{R}^{2} \approx 0.90\right.$ in the slow

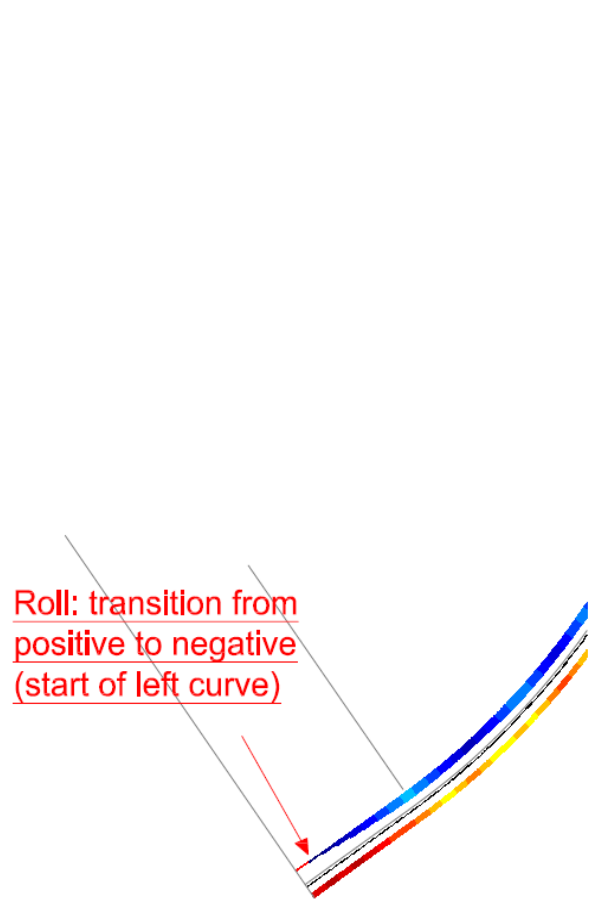

(a)

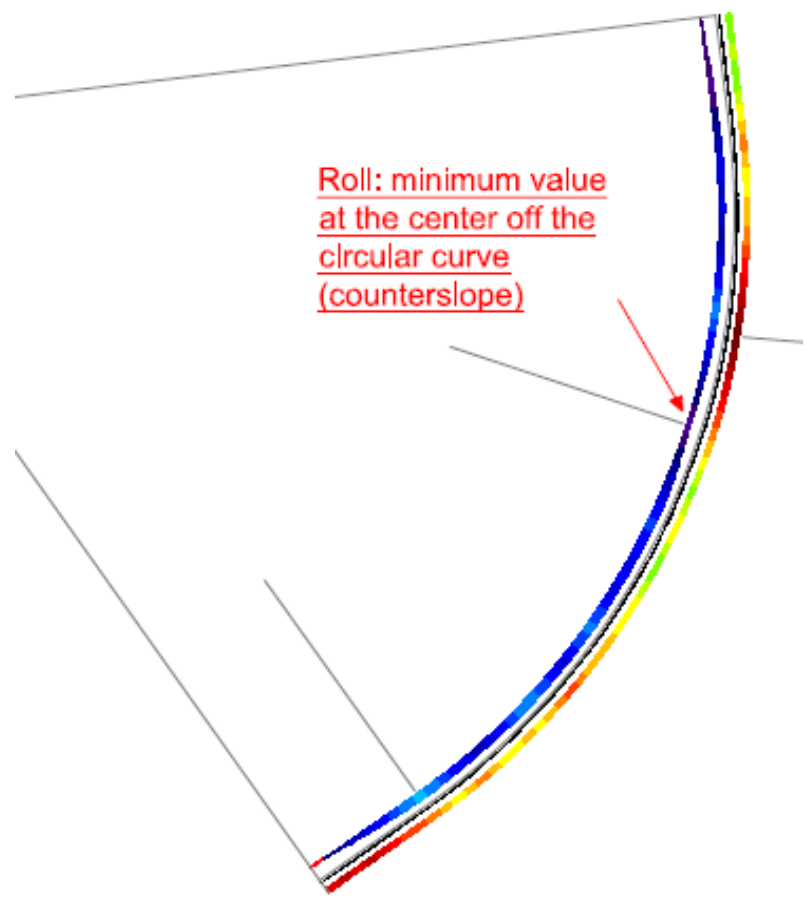

(b)

Fig. 7 Roll-based analysis on tunnel 1 operation from vertical and transverse acceleration: end of straight (a), and counterslope effect (b). 


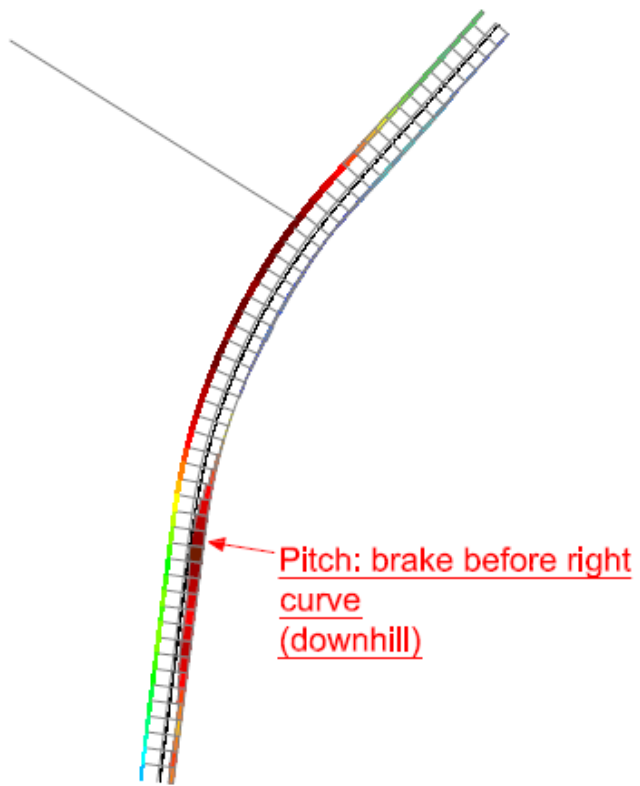

Fig. 8 Pitch-based analysis on tunnel 2: speed reduction at the end of downhill straight.

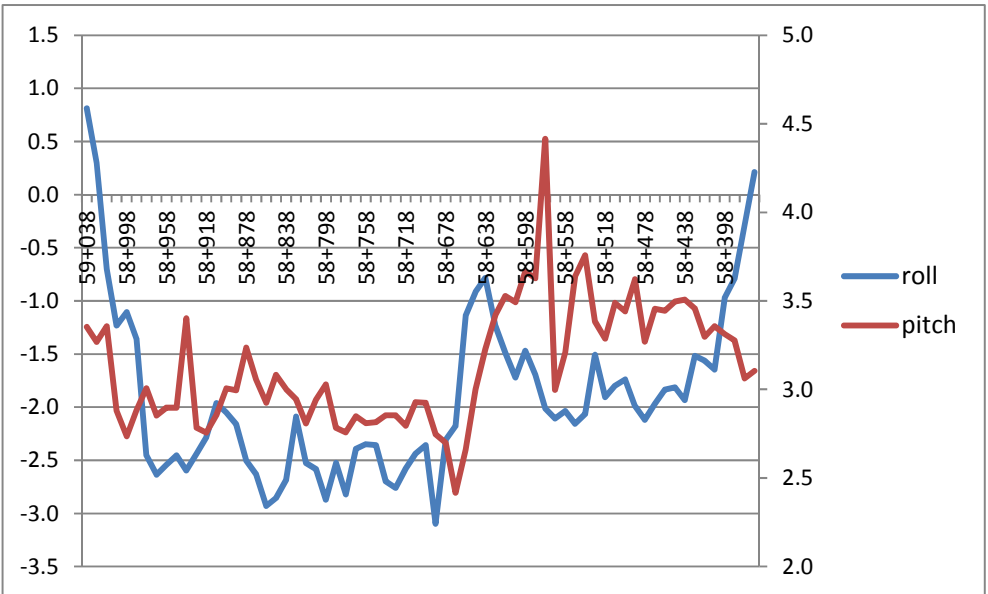

Fig. 9 Pitch vs roll on full test section.

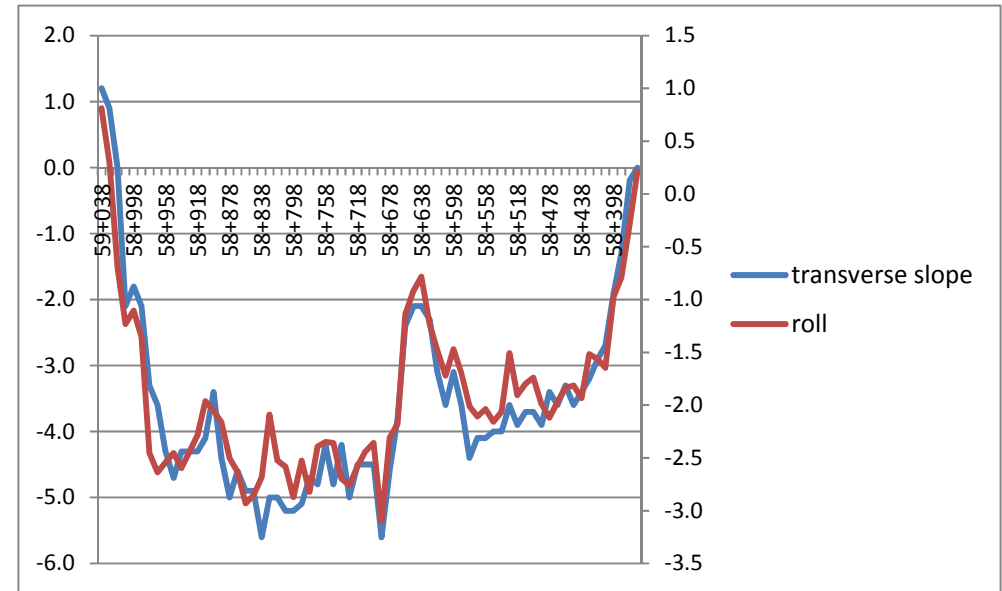

Fig. 10 Transverse slope vs. roll on full test section. 


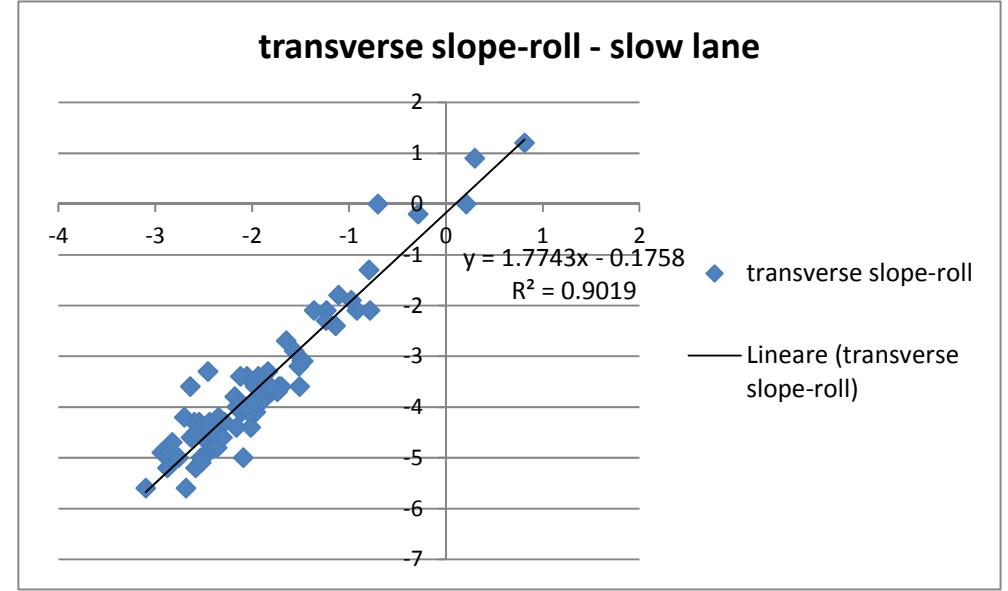

Fig. 11 Correlation function between transverse slope and roll (slow lane).

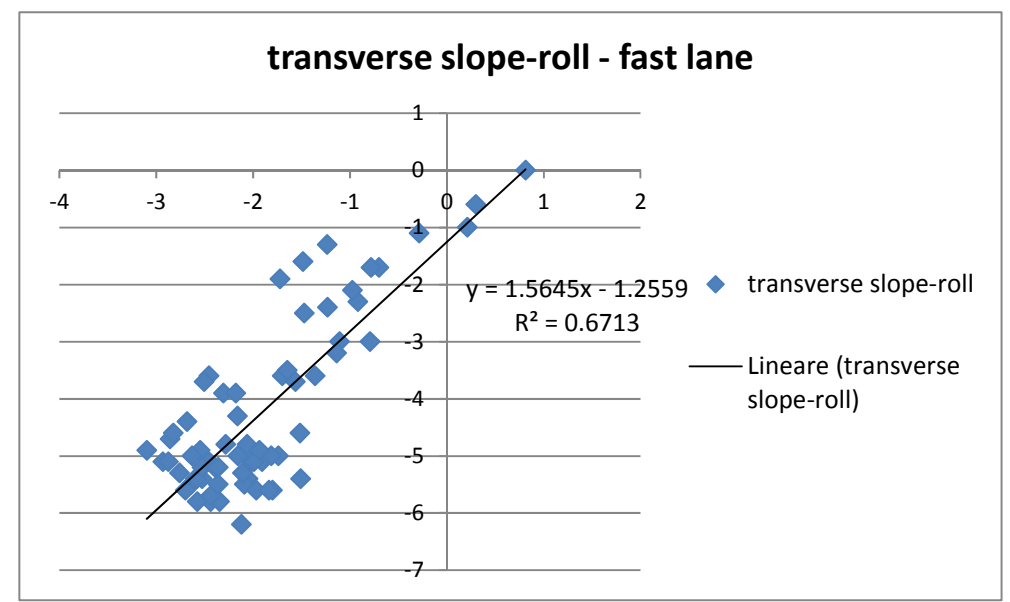

Fig. 12 Correlation function between transverse slope and roll (fast lane).

lane). Instead, vertical accelerations resulted well reflective of slope modifications only in portions of the track which have a regular geometrical asset and in specific transitions from one to another. According to that, it can be assessed that using together vehicle's lateral accelerations and pavement surface's functional characteristics will produce effective results to the goal of highlighting safety risks attributable to the configuration of the carriageway.

\section{Conclusions}

The paper describes the possibility of meeting infrastructure managers' needs by means of high efficiency surveys carried out with the use of an integrated laser system, the LARAN, which combines the Pavemetrics LCMSC with the Optech Lynx Mobile Mapper thus offering high definition 3D images of the infrastructure/environment and of the pavement surface. The availability of information from accessory surveys helps engineers in the trial of realizing a more complete representation of the infrastructure they deal with.

As an example, the results belonging to surveys performed on a portion of an Italian motorway were illustrated and discussed, and the potential of new generation devices has been clearly showed.

In addition, the authors provided some suggestions in order to encourage, on one side, researchers to deepen their knowledge about road quality assurance and focus more and more on safety risks and management, and on the other side infrastructure managers to improve the state of health of the road network they control by constantly monitoring it and by regularly actuating preventive maintenance strategies. 


\section{Acknowledgments}

Authors sincerely want to thank Sina for letting them use (and publish) information being property of one of its customers, and the customer itself for its agreement to the opportunity of the study. In addition, heartfelt thanks go to all of the company staff who permitted to collect the necessary information and to perform the documented surveys with their maximum availability and expertise.

\section{References}

[1] ASTM D5340-12. 2012. "Standard Test Method for Airport Pavement Condition Index Surveys.” ASTM International, 06/01/2012.

[2] ASTM D6433-16. 2016. "Standard Practice for Roads and Parking Lots Pavement Condition Index Surveys." ASTM International, 01/11/2016.

[3] Italian Law. 2001. "Norme funzionali e geometriche per la costruzione delle strade." Ministero delle Infrastrutture e Trasporti-D.M. del 05-11-2001, n. 6792. (in Italian)

[4] Wang, H. 2006. "Road Profiler Performance Evaluation and Accuracy Criteria Analysis." Thesis, Virginia
Polytechnic Institute and State University, Blacksburg, Virginia.

[5] Freitas, E., Pereira, P., Antunes, L., and Domingues, P. 2009. "Study of Data Variability Acquired by Multiple Profilometers." MAIREPAV 6, Turin (Italy), 8-10 July 2009.

[6] Wallman, C. G., and Åström, H. 2001. Friction Measurement Methods and the Correlation between Road Friction and Traffic Safety. A Literature Review. VTI report M911A.

[7] ASTM E1926-08. 2015. "Standard Practice for Computing International Roughness Index of Roads from Longitudinal Profile Measurements.” ASTM International, 05/01/2015.

[8] UNI EN ISO 13473-1:2004: "Characterization of Pavement Texture by Use of Surface Profiles-Part 1: Determination of Mean Profile Depth." Association Francaise de Normalisation.

[9] HD 28/15. DMRB 7.3.1. 2004. "Design Manual for Roads and Bridges, Volume 7, Section 3-Pavement Maintenance Assessment: Skid Resistance.” Highways Agency.

[10] Italian Law. 2011. "Attuazione della direttiva 2008/96/CE sulla gestione della sicurezza delle infrastrutture." Presidente della Repubblica-DL 15-03-2011, n. 35. (in Italian) 